\title{
Tratamiento de salmueras de fermentación de aceitunas verdes
}

\author{
Por A. Garrido Fernández, M. Brenes Balbuena y P. García García \\ Unidad Estructural de Biotecnología de Alimentos. Instituto de la Grasa y sus Derivados. \\ Apartado 1.078. Sevilla.
}

\section{RESUMEN}

Tratamiento de salmueras de fermentación de aceitunas verdes.

El trabajo compara las características de los diversos tratamientos de regeneración aplicables a las salmueras de fermentación de aceitunas verdes estilo sevillano para poderlas reutilizar en otras operaciones dentro del proceso productivo, en especial en el envasado final de los frutos. Con ello, se reduciria el vertido de estas soluciones que son las responsables de la carga contaminante del efluente de estas industrias.

Los dos métodos más interesantes son: adsorción con carbón activo, seguido de una filtración tangencial para separar el absorbente; y la ultrafiltración. En el primer caso, el tipo de carbón activo más eficaz es el denominado GA, que necesita ser utilizado en una proporción entre 5 y $10 \mathrm{~g}$ por litro. En el segundo una membrana de polisulfona de 1.000 daltons de corte molecular es lo más adecuado, trabajando a una presión de 18 bars. Un pretratamiento con bentonita $(2 \mathrm{~g} / 1)$, temperatura entre 30 y $45^{\circ} \mathrm{C}$ y la menor concentración inicial de polifenoles y sólidos en suspensión aumentan el rendimiento de forma considerable. En principio, un estudio económico aproximado de costes indica que la regeneración por cualquiera de los dos sistemas daría una salmuera regenerada a un precio algo inferior al obtenido al preparar el liquido de gobierno para envasar a partir de sus componentes.

PALABRAS-CLAVE: Aceituna verde de mesa - Agua residual - Carbón activo (adsorción sobre) - Salmuera - Ultrafiltración.

\section{SUMMARY}

Treatment for green table olive fermentation brines

The paper studies the characteristics of the several procedures that might be used to regenerate green table olive fermentation brines with the aim of reusing them in other stages of the elaboration process and specially for the final packaging of these fruits. The reuse may considerably reduce the waste water pollution of the canning industry as brines make up about $80 \%$ of the organic pollutant lead of its waste waters.

The two most interesting methods are: adsorption with activated carbon, followed by a cross filtration to separate the adsorbent; and ultrafiltration. The former, should be GA activated carbon, which is the most efficient, in a proportion ranging between 5 and $10 \mathrm{~g}$ per litre. For the second, a polysulphone 1.000 daltons molecular weight cut membrane at 18 bars pressure should be used. A previous treatment of brines with bentonite $(2 \mathrm{~g} / 1)$, a range of temperatures between 30 and $45^{\circ} \mathrm{C}$ as well as low suspended solids and polyphenol contents, increases efficiency notably. In principle, an approximate study of costs shows that regenerated brines are obtained, using either of the two procedures, at slightly lower prices than fresh brines prepared from their ingredients.

KEY-WORDS: Activated carbon (adsorption on) - Brine - Green table olive - Ultrafiltration - Wastewater.

\section{INTRODUCCION}

La producción de aceitunas de mesa en España alcanza habitualmente una cifra en torno a las 250.000 Tm. (C.O.I., 1989). De ellas, $160.000 \mathrm{Tm}$ se preparan como verdes estilo español para cuyo envasado la práctica industrial desecha la salmuera "madre" y la sustituye por otra nueva que se prepara, generalmente, disolviendo en agua, sal, láctico y otros ácidos.

Ello produce, anualmente, alrededor de unos 100.000 $\mathrm{m}^{3}$ de vertidos de salmueras de fermentación, que se arrojan a los sumideros en las instalaciones dedicadas al acondicionamiento de productos finales. Ello contamina gravemente las aguas residuales urbanas o los arroyos y ríos a los que se incorporan.

\subsection{Características de las salmueras de fermen-} tación

En general, son soluciones que llevan una importante carga contaminante medida en términos de $\mathrm{DBO}_{5}$ (Demanda biológica de oxígeno a los cinco días), DQO (Demanda química de oxígeno) y sólidos orgánicos en disolución. Asimismo, contienen una cantidad elevada de cloruro sódico $7-9 \%(p / v)$, y acidez láctica $0,6-1,5 \%(p / v)$, que aseguran la estabilidad del producto durante el período de conservación y que, en principio, podrían ser aprovechables en caso de reutilización. Los niveles entre los que oscilan los diversos parámetros se han recogido en la Tabla I (Garrido, 1990).

Además, conviene resaltar que gran parte de la $\mathrm{DBO}_{5}$ y DQO de las mismas proviene de la presencia del láctico libre y combinado en forma de lejía residual. La proporción concreta que se da en cada caso particular se puede determinar a partir de la Figura 1 (Garrido et al., 1989).

La presencia de polifenoles con estructuras químicas muy estables y muchos de ellos con propiedades bactericidas dificulta la tratabilidad biológica de estos vertidos. También, la alta proporción de cloruro sódico es un inconveniente. Por ello, se requeririan grandes diluciones 
Tabla I

Principales características de las salmueras de aceitunas verdes estilo español o sevillano

\begin{tabular}{|c|c|c|}
\hline \multirow[b]{2}{*}{$\mathrm{pH}$} & \multicolumn{2}{|c|}{ Rango } \\
\hline & 3,6 & 4,3 \\
\hline $\mathrm{NaCl}(\mathrm{g} / \mathrm{l})$ & 60,0 & $-90,0$ \\
\hline Acidez libre (g ac. láctico/l) & 5,0 & $-15,0$ \\
\hline Acidez combinada $(\mathrm{mN})$ & 80,0 & $-120,0$ \\
\hline Polifenoles (g ac. tánico/l) & 1,8 & - 4,0 \\
\hline Color $\left(A_{440}-A_{700}\right)^{*}$ & 0,2 & $-0,6$ \\
\hline Sólidos en suspensión ( $\mathrm{g} / \mathrm{l})$ & 0,2 & $-2,0$ \\
\hline $\mathrm{DBO}_{5}\left(\mathrm{~g} \mathrm{O}_{2} / \mathrm{l}\right)$ & 14,0 & $-18,0$ \\
\hline $\operatorname{coD}\left(\mathrm{g} \mathrm{O}_{2} / 1\right)$ & 16,0 & $-26,0$ \\
\hline
\end{tabular}

* Diferencia de absorbancia a 440 y $770 \mathrm{~nm}$.

Adaptado de: Garrido, 1990.

para adaptarlos a las condiciones exigidas para ser depurados en las plantas de tratamiento convencionales, por lo que las autoridades encargadas de los servicios de saneamiento han presentado siempre objeciones a que las salmueras puedan ser vertidas a los cauces públicos o redes de alcantarillado municipal.

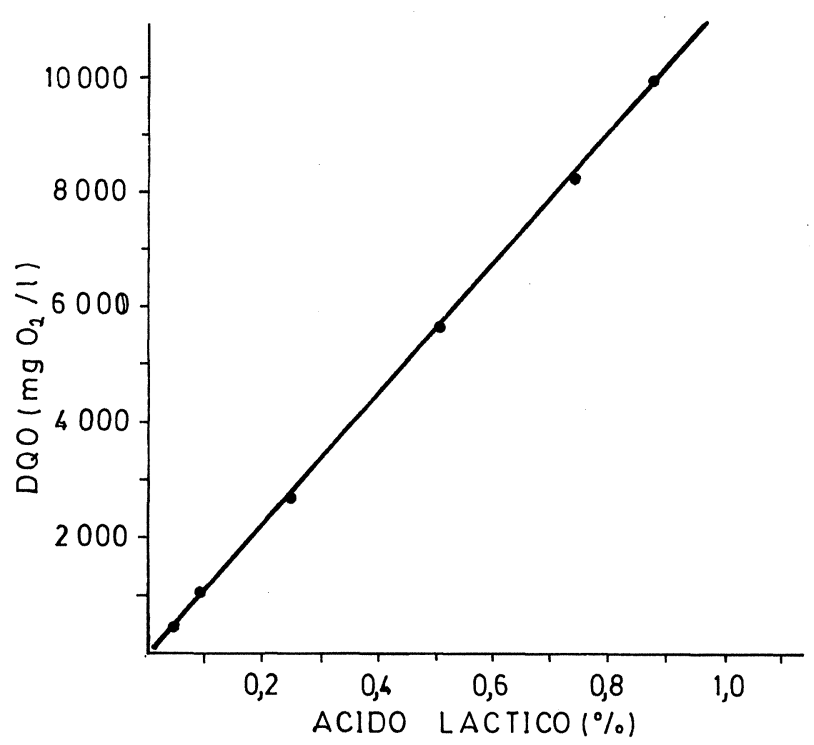

Figura 1

Demanda química de oxígeno ( $\left.\mathrm{mg} \mathrm{O}_{2} / \mathrm{l}\right)$ de soluciones puras de ácido láctico.
1.2. Participación de las salmueras en la carga contaminante generada en las industrias envasadoras

Estas plantas suelen dar lugar a un volumen de vertidos de unos 6-12 l/kg de fruto cuyas características se recogen en la Tabla II (Brenes et al., 1989a). La participación de las salmuera en los mismos se observa en la Tabla III. Representan un volumen reducido y, sin embargo, su contribución a la carga es muy importante. Desde el punto de vista de la tratabilidad general sería conveniente segregarlas y ocuparse de ellas de forma independiente. Con ello, la mayoría de las aguas residuales $(80 \%)$ portaría tan sólo el $30 \%$ de la contaminación, con lo que, posiblemente, con un simple tratamiento físico-químico cumplirian las exigencias de las reglamentaciones que le son aplicables.

Además, si las salmueras se regeneran, la recuperación del ácido y la sal de las mismas, contribuiría a abaratar los costes globales del tratamiento de depuración del conjunto de los vertidos.

Para aplicar esta estrategia es necesario disponer de sistemas de regeneración específicos para salmueras. Este artículo trata de resumir la información disponible al respecto.

Tabla II

Características de los vertidos globales de una industria envasadora de aceitunas verdes

\begin{tabular}{|c|c|c|}
\hline & \multicolumn{2}{|c|}{ Rango } \\
\hline $\mathrm{pH}$ & 3,8 & $-11,5$ \\
\hline Acidez libre (g ac. láctico/l) & 0,0 & - $\quad 1,7$ \\
\hline $\mathrm{NaCl}(\mathrm{g} / \mathrm{l})$ & 3,0 & - 12,0 \\
\hline Sólidos en suspensión $(g / /)^{\star}$ & 0,2 & $-\quad 1,5$ \\
\hline Grasa $(g / l)$ & 0,1 & 1,4 \\
\hline $\mathrm{DQO}^{* *}\left(\mathrm{~g} \mathrm{O}_{2} / \mathrm{l}\right)$ & 0,8 & 5,8 \\
\hline $\mathrm{DBO}_{5}\left(\mathrm{~g} \mathrm{O}_{2} / \mathrm{l}\right)$ & 0,5 & 2,7 \\
\hline
\end{tabular}

- Determinados gravimétricamente con filtros de 0,45 $\mu$.

* Método del dicromato.

Adaptado de: Brenes et al., 1989a.

Tabla III

Desglose de los orígenes de la contaminación global de una fábrica de envasado de aceitunas verdes y el porcentaje del volumen que cada tipo de desecho presenta

\begin{tabular}{lcc}
\hline & $\begin{array}{c}\text { \% de la DQO } \\
\text { total }\end{array}$ & $\begin{array}{c}\% \text { del volumen } \\
\text { total }\end{array}$ \\
\hline Salmueras de fermentación & 70,0 & 22 \\
Lavados de frutos & 17,0 & 45 \\
Limpieza exterior frascos & 0,2 & 11 \\
Otros & 12,3 & 22 \\
\hline
\end{tabular}

Adaptado de: Brenes et al., 1989. 
2. TECNOLOGIAS DISPONIBLES PARA EL TRATAMIENTO DE SALMUERAS DE FERMENTACION DE ACEITUNAS VERDES

\subsection{Tratamientos físico-químicos para su reutili-} zación

Se aplican habitualmente en la fermentación de otros vegetales. Así, McFeecters et al., (1977) pusieron a punto un procedimiento mediante el cual las salmueras de fermentación de pepinillos se neutralizan con $\mathrm{NaOH}$ y después se pasterizan para inhibir las enzimas pectinesterasa y poligalacturonasa que producen ablandamiento en los mismos. Repetidos reusos en sucesivas campañas demostraron su viabilidad.

Un procedimiento alternativo (Geisman y Henne, 1973), consiste en una elevación del pH hasta 11 unidades durante 48 horas, seguido de la subsiguiente corrección con $\mathrm{HCl}$ hasta los niveles adecuados para su adición a los pepinillos. Posteriormente, se ha mejorado la velocidad de sedimentación con la adición de poliectrolito, que reduce el tiempo de la misma a unas 3 horas (Glaros y Geisman, 1980).

También Leoni et al., (1981) han propuesto para las salmueras de aceitunas tratamientos semejantes, si bien eliminaron la pasterización que no era necesaria en este caso. Para obtener una solución suficientemente regenerada sugirieron la adición de polielectrolitos y un tratamiento final con carbón activo. Más tarde, se ha visto, asimismo, que la modificación del $\mathrm{pH}$ de las mismas puede provocar una reducción del $20-40 \%$ en su carga contaminante (García et al., 1990), así como que los procesos de adsorción con resinas intercambiadoras de iones (Brenes y Garrido, 1988a) y, sobre todo, la adsorción con carbón activo (Brenes y Garrido, 1988b), podían ser aplicables con éxito a la eliminación de las sustancias disueltas que contribuían al color. En esta investigación se encontró un tipo de carbón activo que retenía muy selectivamente el color, mientras las pérdidas de ácido láctico se mantenían sólo en valores en torno al 5-10\%. La combinación del mismo con una filtración convencional o tangencial daba lugar a una solución totalmente clarificada que retenía la sal y la casi totalidad de la acidez (Brenes et al., 1990a).

Por otra parte, el empleo de membranas en la preparación de alimentos o en el tratamiento de sus residuos está teniendo una expansión acelerada en productos tales como derivados lácteos (Glover et al., 1978), concentración de zumos (Marson y Moergan, 1968; Peri, 1973), recuperación de proteínas y almidones (Eriksson y von Bockelmann, 1975), tratamiento de salmueras de industrias conserveras de pescado (Welsh y Zade, 1984), filtración y abrillantado de vinos (Chenyom, 1986), etc. La aplicación de las mismas a la regeneración de las salmueras de aceitunas falló en un principio a causa de la especial composición de éstas y de la naturaleza de los elementos filtrantes (Rose, 1982). Sin embargo, la introducción de nuevos materiales ha hecho posible la aplicación de la tecnología de membranas también a dichas soluciones (Brenes et al., 1990b), estudiándose los diferentes factores que influyen en su rendimiento (Brenes et al., 1988) y el efecto en el contenido en polifenoles (Brenes et al., 1990c).

\subsection{Procedimientos de eliminación}

Dadas las dificultades de tratamiento de estas aguas residuales, a lo largo del tiempo se han dado una serie de sugerencias para deshacerse de ellas. Entre las mismas destacan el vertido directo al mar (Kennedy, 1966) o la inyección en capas impermeables en el interior de la tierra, entre 600 y $1.000 \mathrm{~m}$., en zonas donde no existan acuíferos explotables (Batina, 1969). Otra posibilidad es la evaporación de la mayoría del agua que contiene y la destrucción de la materia orgánica obtenida. Sin embargo, dado el carácter corrosivo que comunica la presencia de sal, los sistemas clásicos de evaporadores múltiples se deterioran con gran facilidad. Por ello, Durkee y Lowe (1973) estudiaron la aplicación de la combustión sumergida, en cuyo caso el calentamiento de las salmueras se hizo mediante el paso directo a través de ellas de los gases resultantes de la combustión. La sal se recupera incinerando el concentrado. El sistema es ingenioso, requiere baja inversión inicial y los equipos son de larga duración, aunque el rendimiento energético es inferior al obtenido en los procedimientos convencionales.

De cualquier forma, el sistema más extendido hasta ahora ha sido la evaporación solar, utilizando para ello lagunas de poca profundidad, aproximadamente $80 \mathrm{~cm}$., con o sin aireación. En el caso de aceitunas tipo negras, el propio $\mathrm{pH}$ elevado de las soluciones almacenadas hace inviable el desarrollo de malos olores. El lugar elegido para su ubicación debe estar alejado de las poblaciones, en la dirección opuesta a los vientos dominantes hacia éstas, y favorecer la continua renovación del aire de la superficie. También deben impermeabilizarse para que no contaminen los acuíferos. La utilización de algunos dispositivos para aumentar la capacidad de evaporación puede ser asimismo útil (Heath, 1966).

\section{CRITERIOS PARA LA SELECCION DEL PROCEDI- MIENTO DE REGENERACION}

Dado que las salmueras poseen compuestos de interés, sal y ácido láctico, y que el método de evaporación solar va teniendo una progresiva oposición por parte de vecinos y autoridades, es razonable pensar que la mejor forma de deshacerse de ellas es reutilizarlas. Los posibles tratamientos han ido orientados, fundamentalmente, en la regeneración, bien para su reuso en nuevos procesos fermentativos o en el envasado.

La primera de esas dos alternativas se ha dirigido en especial a productos en los que el acondicionamiento final se hace empleando un ácido diferente al producido en la fermentación como ocurre en los pepinillos, por lo cual la recuperación del acidulante no tenía sentido. Además, en estos procesos no se ha informado de la presencia de inhibidores de las bacterias lácticas ni de que éstas o sus metabolitos interfieran su reuso en posteriores campañas.

La situación con las aceitunas de mesa, por el contra- 
rio, es diferente. En general, las experiencias que se han efectuado hasta ahora de reutilización de salmueras "madres", regeneradas o en diluciones de diverso grado, para posteriores procesos fermentativos no han dado resultados satisfactorios, aunque, últimamente, utilizando una proporción baja de la misma se ha observado una disminución de la alteración denominada "alambrado" en algunas variedades muy sensibles.

Por ello, para este producto los sistemas más adecuados son aquellos que se orientan a la recuperación de dichas soluciones para su reuso, principalmente, en el envasado, así como en otras operaciones en las que se necesite aportación de salmuera, como puede ser el deshuesado, relleno, etc. Sólo en el caso de no aplicar estos tratamientos, o para los residuos de las mismas que queden a pesar de ellos sería necesario eliminarlas.

De acuerdo con todas estas consideraciones, a continuación se va profundizar en las dos tecnologías que parecen las más prometedoras para la recuperación de salmueras.

\section{CARACTERISTICAS DE LA REGENERACION MEDIANTE TRATAMIENTO CON CARBON ACTIVO Y FILTRACION TANGENCIAL}

Una de las principales ventajas que tiene este método es su versatilidad, ya que la intensidad del tratamiento $y$, por tanto, el coste puede ajustarse de acuerdo con la finalidad que se vaya a dar a la salmuera regenerada. Si ésta se destina al envasado, se requerirá la casi total eliminación del color y, en este caso, se deberá recurrir a la utilización de una mayor cantidad de carbón activo. Pero, sin embargo, cuando se vaya a emplear para el "requerido", completar bombonas después de la clasificación, etc., puede ser suficiente sólo una filtración. También, el concentrado pudiera ser fácilmente secado y quemado en la propia industria si esta dispusiera de caldera de orujillo, con lo cual el desprenderse de este residuo resultaría fácil en este caso.

\subsection{Tipo de carbón activo a emplear}

Asimismo, la elección del tipo de carbón activo es decisiva, ya que el comportamiento de los mismos es muy desigual. Como ejemplo, en la Tabla IV se muestran los efectos de diferentes carbones activos, utilizados a dos concentraciones, sobre algunos parámetros de las salmueras. En la misma se aprecian claramente las dos consideraciones anteriores. De los diversos carbones ensayados hasta ahora, sólo el tipo GA, Industrias Kerm, es el que presenta las características más adecuadas, pudiéndose utilizar en una proporción entre 5 y $10 \mathrm{~g} /$, para obtener una regeneración suficiente como para poder destinar el líquido al envasado de frascos. Más detalles sobre el comportamiento del carbón y posterior filtración tangencial a nivel piloto pueden encontrarse en otras publicaciones (Brenes et al., 1988).

\subsection{Efecto en las salmueras y envasados}

El reuso de los líquidos de fermentación a escala de laboratorio y de planta piloto han demostrado que su empleo no produce efectos desfavorables sobre el color ni el sabor del producto (Brenes et al., 1989b). Más recientemente se han efectuado también experiencias a nivel industrial (Garrido et al., 1992).

El tratamiento completo consistió en un proceso de adsorción con carbón, seguido de filtración tangencial a través de un filtro cerámico con un tamaño de poro de 0,2 micras (Chaconsa, S.A., Murcia). La salmuera regenerada se reutilizó en diferentes proporciones, en el líquido de gobierno de los envasados.

El efecto de las diversas proporciones de carbón y diluciones se recoge en la Tabla V. Como puede observarse, el color del líquido de gobierno que se consigue empleando $6 \mathrm{~g} / \mathrm{l}$ de carbón y el $70 \%$ de salmuera madre regenerada es idéntico al obtenido con la blanca. Existe, como es lógico, una elevación de los valores de $\mathrm{pH}$ y lejía residual, que en algunos casos llegan a alcanzar valores excesivamente altos. Ello se debió al hecho de

Tabla IV

Efecto de diferentes carbones activos, utilizados a dos concentraciones, sobre algunas características de las salmueras

\begin{tabular}{|c|c|c|c|c|c|c|c|}
\hline & \multirow{3}{*}{$\begin{array}{c}\text { Salmuera } \\
\text { original }\end{array}$} & \multicolumn{6}{|c|}{ Proporción de carbón activo } \\
\hline & & \multicolumn{3}{|c|}{$5 \mathrm{~g} / \mathrm{l}$} & \multicolumn{3}{|c|}{$10 \mathrm{~g} / \mathrm{l}$} \\
\hline & & $\mathrm{GA}(1)$ & EA & GPG & GA & EA & CPG \\
\hline $\mathrm{pH}$ & 4,20 & 4,20 & 4,25 & 4,23 & 4,24 & 4,32 & 4,30 \\
\hline Acidez libre (g ac. láctico/l) & 7,5 & 7,00 & 7,00 & 6,90 & 6,70 & 6,40 & 6,40 \\
\hline Polifenoles (g ac. tánico/l) & 3,54 & 2,06 & 2,51 & 2,06 & 1,62 & 1,65 & 1,01 \\
\hline Color $\left(A_{440}-A_{700}\right)^{\star}$ & 0,64 & 0,15 & 0,57 & 0,48 & 0,05 & 0,45 & 0,31 \\
\hline
\end{tabular}

(1) Diferentes tipos de carbones activos.

* Diferencia de absorbancia a 440 y $700 \mathrm{~nm}$.

Adaptado de: Brenes y Garrido, 1988b 
partir de una salmuera con valores ya de por sí elevados y a la que, además, no se le habían corregido estos parámetros en el proceso de recuperación. Por tanto, es necesario que dichas soluciones vengan ya con unos niveles de los mismos adecuados o se retoquen en el momento de su tratamiento. Esta operación puede hacerse por los procedimientos habituales y su intensidad dependerá de que el producto se vaya a conservar mediante sus características físico-químicas o por pasterización. Por otra parte, los efectos en el color y textura de los frutos no fueron significativos. Tampoco el análisis sensorial, mediante pruebas de ordenación, puso de manifiesto diferencias estadísticamente apreciables.

\subsection{Evaluación del equipo}

En cuanto al comportamiento del equipo, en la Figura 2 se representa la evolución del flujo medio para una serie de ensayos (Garrido et al., 1992). Para poder recuperar los valores iniciales de caudal de filtrado es imprescindible aplicar correctamente las instrucciones de lavado y regeneración del filtro cerámico, cuyo diámetro interior ( $4 \mathrm{~mm}$ ) sería conveniente que fuera algo mayor para este uso.

\section{CARACTERISTICAS DE LA REGENERACION MEDIANTE ULTRAFILTRACION}

La parte esencial de esta tecnología la constituye, sin duda, la membrana filtrante. Del tamaño del poro depende la capacidad de decoloración, tal como se desprende de la Tabla VI. Para que se consiga una buena clarificación debe emplearse un corte molecular igual o inferior a 1.000 daltons. La composición química de la misma es, asimismo, determinante, pudiéndose afirmar que las de polisulfona no han mostrado dificultades especiales y se han regenerado razonablemente bien a lo largo de las sucesivas experiencias, aunque el tiempo total de estas no ha sido suficiente como para poder sacar conclusiones definitivas sobre la duración previsible de los elementos filtrantes.

\subsection{Condiciones de trabajo}

En el comportamiento de las membranas $y$, sobre todo, en el flujo que se obtiene influyen una serie de variables (Brenes et al., 1988), cuyos efectos resumidos en la Tabla VII, se comentan a continuación:

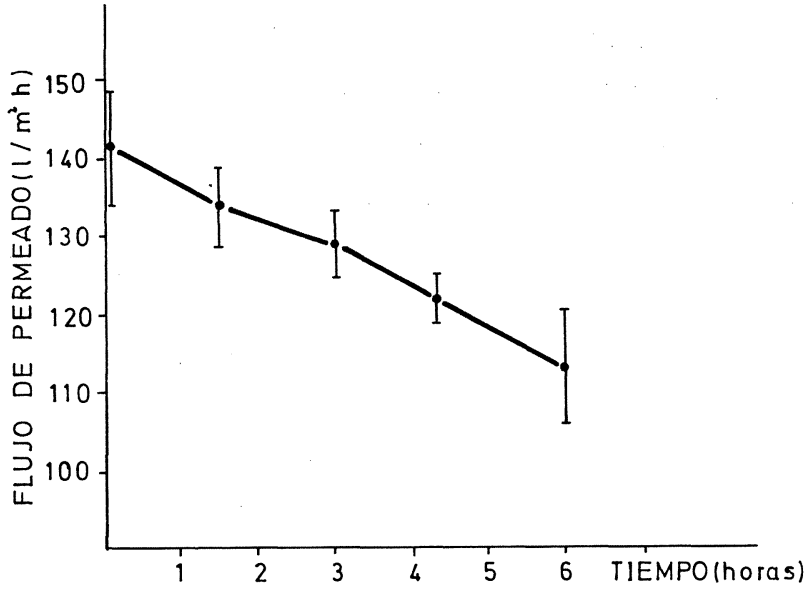

Figura 2

Evolución del flujo de permeado en el equipo de filtración tangencial de salmueras de fermentación de aceitunas verdes estilo español, tratadas con $5 \mathrm{~g} / \mathrm{l}$ de carbón tipo GA.

Tabla V

Efecto en las salmueras de envasado de la reutilización en diversas proporciones de salmueras regeneradas por tratamiento con diferentes porcentajes de carbón activo. Variedad Hojiblanca. Envases de $2.700 \mathrm{~g}$, pasterizados.

\begin{tabular}{|c|c|c|c|c|c|c|c|c|}
\hline & \multicolumn{8}{|c|}{ Tipo de salmuera } \\
\hline & \multicolumn{6}{|c|}{ Regenerada } & \multicolumn{2}{|c|}{ Blanca } \\
\hline & \multicolumn{3}{|c|}{$0,6 \%(1)$} & \multicolumn{3}{|c|}{$0,0 \%$} & \multirow[b]{3}{*}{ Lisa } & \multirow[b]{3}{*}{ Des! } \\
\hline & \multirow{2}{*}{$\frac{70(2)}{\text { Lisa }}$} & \multicolumn{2}{|c|}{35} & \multirow{2}{*}{$\frac{70}{\text { Lisa }}$} & \multicolumn{2}{|c|}{35} & & \\
\hline & & Lisa & Desh & & Lisa & Desh & & \\
\hline $\mathrm{pH}$ & 4,55 & 4,40 & 4,35 & 4,57 & 4,42 & 4,54 & 4,20 & 4,13 \\
\hline $\mathrm{NaCl}(\%, \mathrm{p} / \mathrm{v})$ & 5,90 & 5,60 & 5,60 & 5,90 & 5,70 & 5,80 & 5,70 & 5,60 \\
\hline Acidez libre (g ac. láctico/l) & 5,80 & 5,80 & 5,20 & 6,00 & 5,80 & 5,50 & 5,70 & 5,60 \\
\hline Acidez combinada $(\mathrm{mN})$ & 82,00 & 81,00 & 82,00 & 99,00 & 93,00 & 88,00 & 76,00 & 64,00 \\
\hline Color $\left(A_{440}-A_{700}\right)$ & 0,36 & 0,37 & 0,33 & 0,59 & 0,48 & 0,55 & 0,34 & 0,29 \\
\hline
\end{tabular}

Notas: (1) Proporción de carbón utilizado en la regeneración (p/v).

(2) Proporción de salmuera regenerada en la salmuera de envasado $(\%, p / v)$

Desh $=$ Frutos deshuesados. 
Tabla VI

Efecto del tamaño de poro sobre la eliminación de polifenoles y color en una salmuera de fermentación sometida a ultrafiltración

\begin{tabular}{ccc}
\hline $\begin{array}{c}\text { Tamaño de poro } \\
\text { (daltons) }\end{array}$ & \multicolumn{2}{c}{ Características del permeado } \\
\cline { 2 - 3 } & $\begin{array}{c}\text { Polifenoles } \\
\text { (g ac. tánico/l) }\end{array}$ & $\begin{array}{c}\text { Color } \\
\left(\mathbf{A}_{440}-\mathbf{A}_{\mathbf{7 0 0}}\right)\end{array}$ \\
\hline $10^{6}$ & $5,7^{\star}$ & $0,85^{\star}$ \\
$10^{5}$ & 5,2 & 0,74 \\
$10^{4}$ & 4,3 & 0,26 \\
$10^{3}$ & 3,9 & 0,09 \\
\hline
\end{tabular}

- Valores que se corresponden con los de la salmuera original. Adaptado de: Brenes et al., 1988.

\section{Presión}

El permeado obtenido aumenta con la presión aplicada. En consecuencia, debe operarse a la máxima posible. En este caso se recomienda 18 bars, con lo que aún queda un adecuado margen de seguridad para el equipo.

\section{Concentración inicial de sólidos orgánicos en di-} solución

A medida que la proporción de los mismos se eleva, disminuye el flujo. En la Tabla VII se observa la influencia del contenido de polifenoles, al duplicarse su concentración el caudal obtenido se reduce a la mitad.

\section{Temperatura}

Al subir ésta baja la viscosidad de la solución, por lo que se favorece la velocidad de filtración.

Tabla VII

Efecto de algunas variables sobre el flujo de permeado en la ultrafiltración de salmueras de fermentación a través de una membrana de 1.000 daltons de tamaño de poro.

\begin{tabular}{|c|c|c|c|c|c|}
\hline \multirow[t]{2}{*}{ Variable } & \multicolumn{5}{|c|}{ Evolución del flujo $\left(1 / \mathrm{m}^{2} \mathrm{~h}\right)$ con el tiempo $(\mathrm{h})$} \\
\hline & 1 & 2 & 3 & 4 & 5 \\
\hline \multicolumn{6}{|l|}{ Presión: } \\
\hline 14 bars & 43 & 39 & 37 & 36 & 35 \\
\hline 16 bars & 46 & 44 & 43 & 42 & 42 \\
\hline 18 bars & 55 & 53 & 53 & 52 & 52 \\
\hline \multicolumn{6}{|c|}{ Concentración de polifenoles: } \\
\hline $0,31 \mathrm{~g}$ ac. tánico/l & 55 & 53 & 53 & 52 & 51 \\
\hline $0,61 \mathrm{~g}$ ac. tánico/l & 31 & 30 & 30 & 29 & 28 \\
\hline \multicolumn{6}{|l|}{ Temperatura: } \\
\hline $20^{\circ} \mathrm{C}$ & 48 & 43 & 42 & 40 & 39 \\
\hline $30^{\circ} \mathrm{C}$ & 56 & 54 & 54 & 52 & 51 \\
\hline $40^{\circ} \mathrm{C}$ & 68 & 65 & 63 & 62 & 61 \\
\hline \multicolumn{6}{|c|}{ Tratamientos previos: } \\
\hline Ninguno & 51 & 50 & 50 & 49 & 49 \\
\hline Filtración & 54 & 53 & 53 & 52 & 52 \\
\hline $\begin{array}{l}\text { Floculación } \\
\text { (2 g bentonita/l) }\end{array}$ & 72 & 70 & 70 & 70 & 70 \\
\hline
\end{tabular}

\section{Tratamientos previos}

A medida que la carga de la solución original es mayor, se opone más resistencia a su regeneración. Sin embargo, no todos los pretratamientos han tenido las mismas consecuencias. La filtración sólo consigue una mejora reducida: sin embargo, la floculación con bentonita en una proporción de $2 \mathrm{~g} / \mathrm{l}$ (solución de inbibición del $2-6 \%$ ) favorece de una forma muy sensible el caudal, que pasa casi al doble. Por ello, es de suponer que la actuación de la misma va más allá de la separación de los sólidos en suspensión, si bien, aún no se han estudiado las causas de este efecto.

Todas estas características deben tenerse presentes a la hora de diseñar los tratamientos con los equipos correspondientes, ya que de su optimización dependerá la cuantía de las inversiones iniciales necesarias y el coste de funcionamiento.

\subsection{Efecto en las salmueras y envasados}

El proceso de ultrafiltración con la membrana seleccionada modifica sólo ligeramente las condiciones iniciales de la salmuera, manteniendo bien los porcentajes con $\mathrm{NaCl}$ y de acidez, como puede apreciarse en la $\mathrm{Ta}$ bla VIII. Cuando se trabaja con rechazos finales en torno al $10 \%$, los polifenoles, en general, disminuyen entre el 10 y el $30 \%$. Sin embargo, la eliminación de vanillina, ácido cafeico y ácido $\alpha$-hidroxibenzoico se produce de manera más selectiva y en proporciones más elevadas (55-80\%) (Brenes et al., 1990c).

Experiencias realizadas a nivel industrial han puesto de manifiesto la viabilidad de la tecnología y la posibilidad de utilizar un $45 \%$ de la salmuera así regenerada en el envasado (Garrido et al., 1991).

Como se muestra en la Tabla IX, a estos niveles, a pesar de la acidez combinada original, los valores de $\mathrm{pH}$ son aceptables para un producto pasterizado. Tampoco, en este caso la textura, el color ni las características organolépticas de las aceitunas se modificaron con respecto a los testigos.

\subsection{Evaluación del equipo}

Por lo que respecta al modo de funcionamiento del equipo, no se han encontrado diferencias entre trabajar con sólo recirculación parcial o con esta y reincorporación del líquido filtrado, para mantener constante el volumen de partida.

Según la temperatura, los flujos de permeado pueden oscilar entre $16-18 \mathrm{l} / \mathrm{m}^{2} \mathrm{~h}$, a $16^{\circ} \mathrm{C}$ hasta $22-25 \mathrm{l} / \mathrm{m}^{2} \mathrm{~h}$, a 20 $25^{\circ} \mathrm{C}$, aunque cabe destacar que se han encontrado diferencias notables en el comportamiento de algunas membranas, hechos que la casa fabricante ha asignado a problemas de conservación. Tal como se ha mencionado, estos caudales pueden mejorarse por la adición de bentonita, temperatura de trabajo más elevada, etc.

Como en todos los procesos de membranas, la recuperación del flujo de permeado de unas sesiones a otras depende en gran medida de la correcta realización de la operación de lavado y regeneración de las mismas. En 
Tabla VIII

Influencia de la ultrafiltración en las características físico-químicas de las salmueras de fermentación

\begin{tabular}{lrrr}
\hline Características & \multicolumn{2}{c}{$\begin{array}{c}\text { Salmuera Permeado Concentrado } \\
\text { original }\end{array}$} \\
\hline pH & 3,70 & 3,68 & 3,70 \\
Acidez libre & & & \\
(g ac. láctico/l) & 8,10 & 7,50 & 9,80 \\
Acidez combinada (mN) & 63,00 & 54,00 & 68,00 \\
NaCl (g/l) & 80,00 & 78,00 & 88,00 \\
Polifenoles (g ac. tánico/l) & 2,10 & 1,45 & 4,50 \\
Color $\left(\mathrm{A}_{440}-\mathrm{A}_{700}\right)^{*}$ & 0,44 & 0,06 & $>2,00$ \\
\hline
\end{tabular}

* Diferencia de absorbancia a 440 y $700 \mathrm{~nm}$.

experiencias industriales efectuadas, se ha podido comprobar este hecho, aunque, en general, al cabo del tiempo de funcionamiento estudiado no se ha apreciado pérdida en su capacidad filtrante, utilizando agua como líquido control.

\section{COMPARACION DE LOS ASPECTOS ECONOMI- COS DE LA REGENERACION CON CARBON ACTIVO- FILTRACION TANGENCIAL Y ULTRAFILTRACION}

Está basado en el supuesto de una empresa tipo que prepare unas $40 \mathrm{Tm}$ de aceitunas verdes al día, deseche unos 20.000 I de salmuera y requiera otros 35.000 I para envasar el producto en pequeños recipientes. La producción de líquido regenerado debe ser, entonces, de unos 16.000 l; es decir, unos $900 \mathrm{l} / \mathrm{h}$, si se considera que el equipo filtrante funciona durante 18 horas al dia.

\section{Adsorción-filtración tangencial}

La instalación necesaria debe constar de:

- Silo o tolva de carbón activo, provisto de alimentador continuo de capacidad variable.

- Depósito de fibra de vidrio y resina de poliester para efectuar el contacto salmuera-carbón. Debe ser cerrado y con una boca de hombre para su limpieza. Asimismo, habrá de disponer de un agitador de unas $1.500 \mathrm{r}$ $\mathrm{p} m$ tipo hélice marina y de una serie de embocaduras para entrada de salmuera, retorno de las mismas, etc.

- Filtro doble para retirar los sólidos gruesos en suspensión.

- Equipo de filtración tangencial, con tomas de aire a presión y vapor de agua a 4 y $1,5 \mathrm{~kg} / \mathrm{cm}^{2}$, respectivamente, y una superficie filtrante de unos $4 \mathrm{~m}^{2}$.

Una aproximación al estudio económico de su instalación y funcionamiento se encuentra recogido en la Tabla $X$, considerando que la salmuera se trata con $5 \mathrm{~g} / \mathrm{l}$ de carbón activo.

\section{Ultrafiltración}

La instalación estaría constituida por:

- Un depósito construido en fibra de vidrio y resina de poliester, que, si se trabaja con floculación previa con
Tabla IX

Características de las salmueras de envasado conteniendo el $45 \%$ de salmuera regenerada por ultrafiltración. Envases de $250 \mathrm{~g}$, pasterizados. Variedad Hojiblanca

\begin{tabular}{lrr}
\hline & \multicolumn{2}{c}{ Tipo de salmuera } \\
\cline { 2 - 3 } & Blanca & Regenerada \\
\hline $\mathrm{pH}$ & 3,67 & 4,05 \\
$\mathrm{NaCl}(\mathrm{g} / \mathrm{l})$ & 48,00 & 45,00 \\
Acidez libre (g ac. láctico/l) & 7,60 & 6,90 \\
Acidez combinada $(\mathrm{mN})$ & 65,00 & 100,00 \\
Color $\left(\mathrm{A}_{440}-\mathrm{A}_{700}\right)^{*}$ & 0,07 & 0,09 \\
\hline
\end{tabular}

- Diferencia de absorbancia a 440 y 700 nm. Adaptado de: Garrido, 1991.

bentonita, deberá contar con un agitador de hélice marina. En este caso, también se requiere otro depósito más pequeño para la suspensión de bentonita y su dosificador correspondiente.

- Equipo de ultrafiltración, con una superficie filtrante de unos $42 \mathrm{~m}^{2}$, aproximadamente.

De acuerdo con los datos disponibles, en la Tabla XI, se ha hecho una estimación económica de su coste en base a los mismos supuestos ya mencionados para el otro caso.

Las diferencias que se encuentran entre ambos sistemas se deben fundamentalmente al empleo de carbón activo en el sistema de adsorción-filtración tangencial. En la medida que la proporción del mismo pueda disminuirse lo harán también los gastos correspondientes a este tipo de tratamiento.

Tabla X

Resumen económico de la inversión y coste anual, con un período de amortización de 5 años, del equipo de filtración tangencial de la firma Chaconsa.

Inversión inicial:

Planta de filtración tangencial

Filtro previo

Depósito con agitación

Alimentador de carbón

10.500 .000 ptas 400.000 ptas. 450.000 ptas 800.000 ptas

Total $\quad 12.150 .000$ ptas.

Costo anual:

Amortización (en 5 años) ${ }^{\star} \quad 2.430 .000$ ptas.

Carbón activo

5.720 .000 ptas

Vapor

1.320 .000 ptas.

Energía eléctrica

Mano de obra

740.000 ptas.

660.000 ptas.

Productos para el lavado

50.000 ptas

Total

10.920 .000 ptas

- No se consideran intereses

Adaptado de: Garrido et al., 1991 
Tabla XI

Resumen económico de la inversión y coste anual, con un período de amortización de 5 años, del equipo de ultrafiltración de la firma TFB.

\begin{tabular}{|c|c|}
\hline $\begin{array}{l}\text { Inversión inicial: } \\
\text { Planta de ultrafiltración } \\
\text { Depósito con agitación }\end{array}$ & $\begin{array}{r}18.000 .000 \text { ptas. } \\
450.000 \text { ptas. }\end{array}$ \\
\hline Total & 18.450 .000 ptas. \\
\hline \multicolumn{2}{|l|}{ Costo anual: } \\
\hline Amortización (en 5 años) ${ }^{\star}$ & 3.690 .000 ptas. \\
\hline Membranas & 1.000 .000 ptas. \\
\hline Energía eléctrica & 1.300 .000 ptas. \\
\hline Mano de obra & 270.000 ptas. \\
\hline Productos para el lavado & 40.000 ptas. \\
\hline Total & 6.300 .000 ptas. \\
\hline
\end{tabular}

* No se consideran intereses.

Adaptado de: Garrido et al., 1991.

En el caso de que no se disponga de los equipos de pasterización, deberá considerarse también estas inversiones si se quiere introducir la conservación mediante tratamiento térmico.

Finalmente, se han comparado estos costes con los que se tendrían si se empleara salmuera fresca en el envasado. Sin considerar el agua y estimando que la instalación trabaja 220 días al año y necesita diariamente 35.000 litros de salmuera nueva, dicho gasto podría estimarse en 9.150 .000 ptas., para el ácido láctico $\left(5 \mathrm{~kg} / \mathrm{m}^{3} \mathrm{x}\right.$ $\left.100 / 80 \times 7.700 \mathrm{~m}^{3} \times 190 \mathrm{ptas} / \mathrm{kg}\right)$ y 4.430 .000 ptas. para la sal $\left(50 \mathrm{~kg} / \mathrm{m}^{3} \times 7.700 \mathrm{~m}^{3} \times 11,5 \mathrm{ptas} / \mathrm{kg}\right)$, lo que representa un total de 13.580 .000 ptas. En principio, esta cantidad es superior a la que se requeriría para obtener estas soluciones mediante cualquiera de los procedimientos de regeneración comentados. Además, a esta ventaja sería necesario añadir la reducción de costes que se podrían obtener en el tratamiento del vertido final menos contaminado.

Con ello se abre la posibilidad de que dichos sistemas se introduzcan como un paso más en la estrategia del tratamiento global de los residuos líquidos de las industrias envasadoras de aceitunas verdes, en su camino de aproximación al cumplimiento de la legislación sobre aguas residuales que le es aplicable.

\section{BIBLIOGRAFIA}

Batina, R.T.; McNeill, L. (1969).- "Practical approaches to cannery waste treatment problems".- 48th Annual Technical Report of California Olive Association, Monterrey (USA), 67-72.

Brenes, M.; Garcia, P. y Garrido, A. (1988).- "Regeneration of spanish style green olives brines by ultrafiltration".- J. Food Sci. 53, 17331735 .

Brenes, M. y Garrido, A. (1988a).- "Regeneración de salmueras de aceitunas verdes estilo español con resinas intercambiadoras de iones".Grasas y Aceites 39, 22-27.

Brenes, M. y Garrido, A. (1988c).- "Regeneración de salmueras de aceitunas verdes estilo español con carbón activo y tierras decolorantes".- Grasas y Aceites 39, 96-101.
Brenes, M.; de Vicente, J.; García, P. y Garrido, A. (1989a).- "Caracteristicas de las aguas residuales generadas en las aceitunas de mesa".Grasas y Aceites 40, 287-289.

Brenes, M.; Garcia, P. y Garrido, A. (1989b).- "Influencia en el envasado de aceitunas verdes estilo español del reuso de salmueras regeneradas".- Grasas y Aceites 40, 182-199.

Brenes, M.; Sánchez, F.; García, P. y Garrido, A. (1990b).- "Procedimiento de regeneración de salmueras de aceitunas y otros productos vegetales para su utilización posterior".- Patente Es. n. ${ }^{2} 2016470$ (1-11-90).

Brenes, M.; Sánchez, F.; García, P. y Garrido, A. (1990c).- "Procedimiento para la regeneración de salmueras de fermentación de aceitunas verdes estilo español mediante ultrafiltración-ósmosis inversa y floculación previa con bentonita".- Patente Es. n.? 2016471. (1-11-90).

Brenes, M.; Montaño, A. y Garrido, A. (1990d).- "Ultrafiltration of green table olives brines: Influence of some operating parameters and effect on polyphenol composition".- J. Food Sci. 55, 214-217.

COI. Consejo Oleicola Internacional (1989).- "Balance de la producción de aceitunas de mesa".- Comité de Expertos del COl. Madrid, noviembre.

Cheryan, M. (1986).- "Ultrafiltration handbook".- Technomic Publishing Company. Inc., Philadelphia, U.S.A.

Durkee, E.L. y Lowe, E. (1973).- "Field test of salt recovery system for spent pickle brine".- J. Food Sci. 38, 507-511.

Ericksson, G. y von Bickelmann, Y. (1975) .- "Ultrafiltration al animal blood serum".- Technol. Microbiol. Proc. Biochem. 9, 11-15

Garcia, P.; Brenes, M.; de Vicente, J. y Garrido, A. (1990).- "Depuración de las aguas residuales de las plantas envasadoras de aceitunas verdes mediante tratamientos fisico-químicos".- Grasas y Aceites 41, 263-269.

Garrido, A.; Garcia, P.; Rejano, L.; Brenes, M.; Sánchez, A.H. y de Vicente, J. (1989).- "Caracterización de las aguas residuales de las industrias envasadoras de aceitunas. Estudio y aplicación de sistemas de depuración".- Informe final del contrato de investigación con ACEMESA.

Garrido, A.- "Environmental impact. Fermented fruits and vegetables".Mesa Redonda I Congreso Internacional de Tecnología y Desarrollo Alimentario. Murcia, 13-16. Noviembre, 1990. Proceedings en prensa.

Garrido, A.; Garcia, P.; Rejano, L.; Brenes, M. y Sánchez, A.H. (1991)."Regeneración de salmueras de aceitunas de mesa".- Informe final. Programa PROA. C.S.I.C. Sevilla.

Garrido, A.; Brenes, M. y Garcia, P. (1992).- "Field tests of table olive brine recycling by adsorption with activated charcoal and ultrafiltration".- J. Food. Eng. 17, 291-305.

Geisman, J.R. y Henne, R.E. (1973).- "Recycling food brine eliminates pollution".- Food Eng. 45, 119-123.

Glaros, T. y Heisman, J.R. (1980).- "Organic floculants spedd spist pickle curing brine recycling".- J. Food Sci. 45, 402-403.

Glover, F.A.; S. Skudder; P.J.; Stothart, P.H. y Evans, E.W. (1978)."Reviews of the progress of dairy science: reverse osmosis and ultrafiltration in dairying".- J. Dairy Res. 45, 291-318.

Heath, D.M. (1966).- "Reclamation and renovation possibilities for salt contamined wastes".- 45th Annual Technical Report of the California Olive Association, Monterrey (USA), 50-55.

Kennedy, R.M. (1969).- "Panel discusión on waste and water problems".45th Annual Technical Report of California Olive Association, Monterrey (USA), 13-19.

Leoni, C.; Grischott, F. y Fortini, G. (1981).- "Trattamento di effluenti specifici dell'industria alimentari particolarmente ricchi di sustanze organichi".- Ind. Conserve 56, 173-177.

McFeeters, R.F.; Palmitkar, M.P.; Veltine, M.; Fehringer, N. y Coon (1977)."Reuse of brines in commercial cucumber fermentation".- Inc. Proc. 8th Natl. Simp. Food Process Wastes. US. Envirom. Agency 600/277-84.

Marson, R.L. y Morgan, A.J. (1968).- "Juice concentration by reverse osmosis".- Food Technol. 22, 631-634

Peri, C. (1973).- "Orange juice concentration reverse osmosis retention. Permeation rate and economic of the process.- "Int. Fructsaf. Wiss. Tech. Kimm. Lrelin 13, 119-125.

Rose, W.W. (1982).- "Innovative treatment technology".- 59th Annual Technical Report of the California Olive Association, 49-58.

Welsh, F. y Zall, R. (1984).- "An ultrafiltration activated carbon treatment system for renovating fishery refrigeration brines".- Can. Inst. Food Sci. Technol. J. 17, 92-96.

(Recibido: Enero 1992) 